\begin{tabular}{|c|l|}
\hline Title & Molecular dynamics simulations of nucleation from vapor to solid composed of Lennard-Jones molecules \\
\hline Author(s) & Tanaka, Kyoko K.; Tanaka, Hidekazu; Y amamoto, Tetsuo; Kawamura, Katsuy uki \\
\hline Citation & $\begin{array}{l}\text { Journal of Chemical Physics, 134(20), 204313 } \\
\text { https://doi.org/10.1063/13593459 }\end{array}$ \\
\hline Issue Date & 2011-05-28 \\
\hline Doc URL & http://hdl.handle.net/2115/45807 \\
\hline Rights & $\begin{array}{l}\text { Copyright 2011 A merican Institute of Physics. This article may be downloaded for personal use only. A ny other use } \\
\text { requires prior permission of the author and the A merican Institute of Physics. The following article appeared in J. } \\
\text { Chem. Phys. 134, 204313(2011) and may be found at https:/dx.doi.org/10.1063/.3593459 }\end{array}$ \\
\hline Type & article \\
\hline File Information & JCP134 20_204313.pdf \\
\hline
\end{tabular}

Instructions for use 


\title{
Molecular dynamics simulations of nucleation from vapor to solid composed of Lennard-Jones molecules
}

\author{
Kyoko K. Tanaka, ${ }^{1, a)}$ Hidekazu Tanaka, ${ }^{1}$ Tetsuo Yamamoto, ${ }^{1}$ and Katsuyuki Kawamura ${ }^{2}$ \\ ${ }^{1}$ Institute of Low Temperature Science, Hokkaido University, Sapporo 060-0819, Japan \\ ${ }^{2}$ Graduate School of Environmental Science, Okayama University, Okayama 700-8530, Japan
}

(Received 28 February 2011; accepted 4 May 2011; published online 27 May 2011)

\begin{abstract}
We performed molecular dynamics (MD) simulations of nucleation from vapor at temperatures below the triple point for systems consisting of $10^{4}-10^{5}$ Lennard-Jones (L-J) type molecules in order to test nucleation theories at relatively low temperatures. Simulations are performed for a wide range of initial supersaturation ratio $\left(S_{0} \simeq 10-10^{8}\right)$ and temperature $(k T=0.2-0.6 \varepsilon)$, where $\varepsilon$ and $k$ are the depth of the L-J potential and the Boltzmann constant, respectively. Clusters are nucleated as supercooled liquid droplets because of their small size. Crystallization of the supercooled liquid nuclei is observed after their growth slows. The classical nucleation theory (CNT) significantly underestimates the nucleation rates (or the number density of critical clusters) in the low- $T$ region. The semi-phenomenological (SP) model, which corrects the CNT prediction of the formation energy of clusters using the second virial coefficient of a vapor, reproduces the nucleation rate and the cluster size distributions with good accuracy in the low- $T$ region, as well as in the higher- $T$ cases considered in our previous study. The sticking probability of vapor molecules onto the clusters is also obtained in the present MD simulations. Using the obtained values of sticking probability in the SP model, we can further refine the accuracy of the SP model. () 2011 American Institute of Physics. [doi:10.1063/1.3593459]
\end{abstract}

\section{INTRODUCTION}

Nucleation from the vapor phase plays a very important role in many areas of science and technology. The classical nucleation theory (CNT) is a model most widely used for describing homogeneous nucleation and provides the nucleation rate as a function of ambient vapor properties. ${ }^{1-4}$ However, several studies have reported that the CNT fails to explain results obtained in laboratory experiments. ${ }^{5-18}$ In addition to laboratory experiments, numerical approaches such as molecular dynamics (MD) or Monte Carlo (MC) simulations are powerful methods by which to investigate a homogeneous nucleation process. The MD and MC simulations also showed that the nucleation rates obtained by MD and MC simulations are significantly different from predictions by the CNT. ${ }^{19-37}$ The nucleation rate is determined by the number density (or the work of formation) of the critical clusters, which are thermodynamically the largest unstable clusters. Although the critical clusters are nano-sized in the parameter range of nucleation experiments, the CNT estimates the work of formation of such small critical clusters, by simply using the surface tension of bulk material. This oversimplification in the CNT causes its inaccuracy.

During the last two decades, there have been significant advances in theoretical models of homogeneous nucleation. ${ }^{8-10,13,38-50}$ One of the most successful and useful models is the semi-phenomenological (SP) model proposed by Dillmann and Meier. ${ }^{8}$ The SP model corrects the evaluation of the formation energy of clusters in the CNT by using

${ }^{a)}$ Electronic mail: kktanaka@lowtem.hokudai.ac.jp. the second virial coefficient of a vapor. The predictions of the SP model agree surprisingly well with the experimental data for water, nonane, and $n$-alcohols. ${ }^{8,10-14}$ Although an inconsistency in Dillmann and Meier's original model was pointed out by Delale and Meier ${ }^{10}$ and by Laaksonen et al. ${ }^{13}$ this inconsistency has been overcome by them without spoiling the agreement with the experimental results. By performing MD simulations of nucleation for Lennard-Jones (L-J) type molecules at temperatures higher than or nearly equal to the triple point, Tanaka et $a l .{ }^{36}$ tested the SP model and found that the SP model also provides excellent agreement with their MD simulations. Because of its phenomenological property, however, the parameter range and types of material for which the SP model is applicable has not yet been clarified. As such, further tests on the SP model are necessary. Nucleation theories involving a concept of non-sharp boundary between clusters and vapor have been also developed. These include the density functional theory (DFT) (Refs. 38 and 39) and the extended modified liquid drop model-dynamical nucleation theory. ${ }^{40,41}$ Kalikmanov et al. ${ }^{47}$ and Napari et al. ${ }^{50}$ showed that these theories perform better than the classical nucleation theory by the comparison to the MD simulation data.

The scaling law proposed by McGraw and Laaksonen $^{38,39}$ provided a different correction to the CNT. In their scaling law, the corrected formation energies for each cluster are given by a simple offset from the CNT prediction. The offset is a function of temperature only and is independent of the cluster size, whereas the energy correction in the SP model is dependent on the size. Their scaling law was supported by the DFT (Refs. 38 and 39) and was confirmed by MC simulations for clusters larger than 
a certain threshold size. ${ }^{22,31,45,46,48,49}$ These results suggest that the SP model is inapplicable, at least, when the critical cluster is larger than the threshold size.

In the present paper, we performed a large number of MD simulations of homogeneous nucleation for Lennard-Jones type molecules at temperatures below the triple point, where the vapor finally condenses to a solid phase. The vapor number density (or the supersaturation ratio) is another parameter. For various number densities, MD simulations are carried out at each temperature. Through comparison with the nucleation rates and the cluster size distributions obtained from our MD simulations, we investigate the validity of the SP model in such a low-temperature region. Our MD simulations are also able to clarify when clusters are crystallized during their growth.

Furthermore, the sticking probability $\alpha$ of vapor molecules onto clusters can be examined in MD simulations, by observing the growth rate of stable clusters larger than the critical size. Although the nucleation rate is proportional to $\alpha$ in the nucleation theory, the sticking probability is usually assumed to be unity. This would cause another type of error in the nucleation theory. Some authors have examined the sticking probability onto the planar liquid surface, ${ }^{51,52}$ which may have a different value for small clusters. In Sec. II, we briefly describe the CNT and the SP model. We will also describe the relation between the nucleation theory and the cluster expansion theory. ${ }^{53}$ In Sec. III, the numerical procedure of our MD simulation is described, and in Sec. IV, we present our numerical results. Finally, in Sec. V, we summarize the results of the present study.

\section{NUCLEATION THEORY}

\section{A. Nucleation rate and size distribution of clusters}

In the homogeneous nucleation theory, the nucleation rate $J$ (i.e., the net number of nucleated grains per unit volume and time) is expressed as ${ }^{4,9}$

$$
J=\left\{\sum_{i=1}^{\infty} \frac{1}{R^{+}(i) n_{e}(i)}\right\}^{-1},
$$

where $R^{+}(i)$ is the transition rate of an $i$-mer (which is a cluster containing $i$ molecules) to an $(i+1)$-mer, and $n_{e}(i)$ is the equilibrium number density of $i$-mers. For monomers (i.e., vapor molecules), we set $n_{e}(1)=n(1)$. The transition rate $R^{+}(i)$ is given by

$$
R^{+}(i)=\alpha n(1) v_{\mathrm{th}}\left(4 \pi r_{0}^{2} i^{2 / 3}\right),
$$

where $v_{\text {th }}$ is the thermal velocity given by $\sqrt{k T / 2 \pi m}$, and $\alpha$ is the sticking probability. Usually, $\alpha$ is simply assumed to be unity. Furthermore, the radius of a monomer $r_{0}$ is defined by $\left(3 m / 4 \pi \rho_{m}\right)^{1 / 3}$ with the mass of a molecule $m$ and the bulk density $\rho_{m}$. Thus, $4 \pi r_{0}^{2} i^{2 / 3}$ in Eq. (2) represents the surface area of the $i$-mer.

In order to calculate the nucleation rate from Eq. (1), we need an expression of the equilibrium number density of $i$ mers, which is obtained from the equilibrium condition

$$
\mu_{i}=i \mu_{1}
$$

where $\mu_{i}$ and $\mu_{1}$ are the chemical potentials of $i$-mers and vapor, respectively. These chemical potentials can be expressed as

$$
\mu_{i}\left(T, P_{i}\right)=k T \ln \left(\frac{P_{i}}{P_{0}}\right)+\mu_{i}^{0}(T) \quad(i \geq 1),
$$

where $\mu_{i}^{0}(T)$ and $\mu_{1}^{0}(T)$ are the chemical potentials of $i$-mers and vapor at the standard pressure $P_{0}$, and $P_{i}$ is the partial pressure of $i$-mers (or vapor for $i=1$ ). Substituting Eq. (4) into Eq. (3), for $n_{e}(i)$, we have

$$
n_{e}(i)=\frac{P_{0}}{k T} \exp \left\{i \ln \left(\frac{P_{1}}{P_{0}}\right)-\frac{\mu_{i}^{0}-i \mu_{1}^{0}}{k T}\right\},
$$

where we used $P_{i}=n_{e}(i) k T$. The saturated vapor pressure $P_{\text {sat }}(T)$ is given by

$$
\frac{P_{\text {sat }}}{P_{0}}=\exp \left(\frac{\mu_{\text {sat }}-\mu_{1}^{0}}{k T}\right),
$$

where $\mu_{\text {sat }}(T)$ is the chemical potential of a saturated vapor. Using this equation, we can rewrite Eq. (5) as follows:

$$
n_{\mathrm{e}}(i)=\frac{P_{0}}{k T} \exp \left(i \ln S-\frac{\mu_{i}^{0}-i \mu_{\mathrm{sat}}}{k T}\right),
$$

where $S$ is the supersaturation ratio defined by

$$
S=P_{1} / P_{\text {sat }} .
$$

Note that the supersaturation ratio is defined with the partial pressure of monomers instead of the total pressure. ${ }^{44}$

The free energy (or the work), $\Delta G_{i}$, associated with forming an $i$-mer cluster from $i$ individual molecules is defined by

$$
\Delta G_{i} \equiv \mu_{i}\left(T, P_{i}=P_{1}\right)-i \mu_{1}\left(T, P_{1}\right) .
$$

Using Eqs. (4) and (6), the free energy is rewritten as

$$
\Delta G_{i}=k T \ln \left(\frac{P_{\mathrm{sat}}}{P_{0}}\right)-(i-1) k T \ln S+\mu_{i}^{0}-i \mu_{\mathrm{sat}}
$$

and we obtain the following well-known expression for the equilibrium number density:

$$
n_{\mathrm{e}}(i)=\frac{P_{1}}{k T} \exp \left\{-\frac{\Delta G_{i}}{k T}\right\} .
$$

The free energy $\Delta G_{i}$ attains a maximum value for clusters of a certain size $i^{*}$. Such clusters are referred to as critical clusters. Clusters larger than $i^{*}$ are stable and continue to grow whereas those smaller than $i^{*}$ are unstable.

Since the number density of critical clusters is usually extremely low due to their large $\Delta G_{i}$, it regulates the nucleation rate given by Eq. (1). If a peak of $\Delta G_{i}$ is sharp enough and the size $i^{*}$ are sufficiently large, the summation in Eq. (1) can be replaced with an integration and we have

$$
J=R^{+}\left(i^{*}\right) n_{\mathrm{e}}\left(i^{*}\right) Z,
$$

where the Zeldovich factor $Z$ is given by

$$
Z=\sqrt{\frac{1}{2 \pi k T}\left[-\frac{d^{2} \Delta G_{i}}{d i^{2}}\right]_{i=i^{*}} .}
$$


In the quasi-steady state of nucleation, the number densities of various $i$-mer clusters deviate from the equilibrium values $n_{\mathrm{e}}(i)$. Actual number densities $n(i)$ are expressed in terms of $n_{\mathrm{e}}(i)$ as follows:

$$
n(i)=J n_{\mathrm{e}}(i) \sum_{j=i}^{\infty} \frac{1}{R^{+}(j) n_{\mathrm{e}}(j)} .
$$

Deviation of the number density in supersaturation from that in equilibrium is significant for stable clusters larger than the critical size $i^{*}$. This expression for the cluster size distribution will be used for the comparison between the MD simulations and the theoretical models.

As shown above, the nucleation rate and the cluster size distribution are determined by the chemical potentials of clusters. On the other hand, the thermodynamics of $i$-mer clusters in an imperfect gas is described by cluster integrals in the cluster expansion theory. ${ }^{53}$ According to the cluster expansion theory, we obtain the following relation between the chemical potential $\mu_{i}^{0}(T)$ and the cluster integral $b_{i}(T)$ (see Appendix A):

$$
\mu_{i}^{0}-i \mu_{\mathrm{sat}}=-k T \ln \left\{b_{i}\left(\frac{P_{\mathrm{sat}}}{k T}\right)^{i-1} \frac{P_{\mathrm{sat}}}{P_{0}}\right\} .
$$

Substituting Eq. (15) into Eq. (10) gives the following relation between $\Delta G_{i}$ and $b_{i}$ :

$$
\Delta G_{i} / k T=-(i-1) \ln S-\ln \left\{b_{i}\left(\frac{P_{\text {sat }}}{k T}\right)^{i-1}\right\} .
$$

This expression for $\Delta G_{i}$ is identical to that derived by Merikanto et al. ${ }^{44}$

\section{B. Theoretical models for $\mu_{i}^{0}$ and $\Delta G_{i}$}

In the classical nucleation theory, the free energy of formation $\Delta G_{i, \mathrm{CNT}}$ is given by

$$
\Delta G_{i, \mathrm{CNT}} / k T=-i \ln S+\eta i^{2 / 3},
$$

where

$$
\eta=4 \pi r_{0}^{2} \gamma / k T
$$

and $\gamma$ is the surface tension of the condensed phase. Then, the chemical potential of an $i$-mer at a standard pressure $\mu_{i, \mathrm{CNT}}^{0}$ is given by

$$
\mu_{i, \mathrm{CNT}}^{0}=i \mu_{\mathrm{sat}}+k T\left\{-\ln \left(\frac{P_{\mathrm{sat}} S}{P_{0}}\right)+\eta i^{2 / 3}\right\} .
$$

Using these expressions, the equilibrium number density $n_{e}(i)$ and the nucleation rate $J$ can be obtained.

In the classical theory, the above expressions contain two theoretical inconsistencies. The first is the $S$-dependence of $\Delta G_{i}$ and $\mu_{i}^{0}$. Based on the definition, the chemical potential at the standard pressure $\mu_{i}^{0}$ should be independent of $S$ (or $\left.P_{1}\right)$. However, this is not the case for Eq. (19). This is because the $S$-dependence of $\Delta G_{i, \mathrm{CNT}}$ does not agree with Eq. (10). The second inconsistency is related to the values of $\Delta G_{i}$ and $\mu_{i}^{0}$ at $i=1$. In the above expressions, for $i=1, \Delta G_{i, \mathrm{CNT}}$ does not vanish and $\mu_{i, \mathrm{CNT}}^{0}$ is not equal to $\mu_{1}^{0}$. In order to resolve these problems, a simple modification of the classical theory has been proposed in a number of studies. ${ }^{9,17}$ In the modified CNT (MCNT), $\Delta G_{i, \mathrm{MCNT}}$ and $\mu_{i, \mathrm{MCNT}}^{0}$ are given by

$$
\Delta G_{i, \mathrm{MCNT}} / k T=-(i-1) \ln S+\eta\left(i^{2 / 3}-1\right)
$$

and

$$
\mu_{i, \mathrm{MCNT}}^{0}=i \mu_{\mathrm{sat}}+k T\left\{-\ln \left(\frac{P_{\mathrm{sat}}}{P_{0}}\right)+\eta\left(i^{2 / 3}-1\right)\right\},
$$

respectively. Although these modifications resolve the theoretical inconsistencies, the modified-CNT does not succeed in explaining the experimental data (or the numerical data) of the nucleation rate. ${ }^{17,36}$

In the semi-phenomenological model, another term is added to $\mu_{i, \mathrm{MCNT}}^{0}$ (or $\Delta G_{i, \mathrm{MCNT}}$ ). The free energy of formation $\Delta G_{i, \mathrm{SP}}$ and the chemical potential of an $i$-mer at the standard pressure $\mu_{i, \mathrm{SP}}^{0}$ in the SP model are given by

$$
\Delta G_{i, \mathrm{SP}} / k T=-(i-1) \ln S+\eta\left(i^{2 / 3}-1\right)+\xi\left(i^{1 / 3}-1\right)
$$

and

$$
\begin{aligned}
\mu_{i, \mathrm{SP}}^{0}= & i \mu_{\mathrm{sat}}+k T \\
& \times\left\{-\ln \left(\frac{P_{\mathrm{sat}}}{P_{0}}\right)+\eta\left(i^{2 / 3}-1\right)+\xi\left(i^{1 / 3}-1\right)\right\},
\end{aligned}
$$

where $\xi(T)$ is a non-dimensional parameter depending on $T$, which is fixed by the use of the second virial coefficient. Using the second virial coefficient $B_{2}\left(=-b_{2}\right)$, the formation energy of a dimer $\Delta G_{2}$ is given by $-k T \ln \left(-B_{2} P_{1} / k T\right)$ (see Eq. (16)). Assuming that this is satisfied in Eq. (22) for $i=2$, we can fix the parameter $\xi$ as

$$
\xi=-\frac{1}{2^{1 / 3}-1}\left[\ln \left(\frac{-B_{2} P_{\text {sat }}}{k T}\right)+\left(2^{2 / 3}-1\right) \eta\right] .
$$

Then, the equilibrium number density $n_{e}(i)$ and the cluster integral $b_{i}$ are given in the SP model by

$$
n_{\mathrm{e}}(i)=\frac{P_{1}}{k T} S^{i-1} \exp \left\{-\eta\left(i^{2 / 3}-1\right)-\xi\left(i^{1 / 3}-1\right)\right\}
$$

and

$b_{i}=\exp \left\{-(i-1) \ln \left(\frac{P_{\text {sat }}}{k T}\right)-\eta\left(i^{2 / 3}-1\right)-\xi\left(i^{1 / 3}-1\right)\right\}$.

By setting $\xi=0$, we also obtain expressions of $n_{\mathrm{e}}(i)$ and $b_{i}$ in the modified-CNT.

\section{NUMERICAL PROCEDURE}

With MD simulations, we directly observe the nucleation process in systems of $10^{4}$ to $2 \times 10^{5}$ Lennard-Jones type molecules. The interaction potential between two LennardJones type molecules, $u_{i j}(r)$, is given by

$$
u_{i j}(r)=4 \varepsilon\left[\left(\frac{\sigma}{r}\right)^{12}-\left(\frac{\sigma}{r}\right)^{6}\right]
$$


TABLE I. Parameters in the MD simulations. $T$ : temperature, $N$ : particle number, $L$ : cell size, $n(1)_{t=0}$ : initial number density of monomers, $t_{\mathrm{end}}$ : simulation time, and $S_{0}$ : initial supersaturation ratio. L-J units are used.

\begin{tabular}{lcrrrcc}
\hline \hline Run\# & $T[\varepsilon / k]$ & $N$ & $L[\sigma]$ & $n(1)_{t=0}\left[\sigma^{-3}\right]$ & $t_{\text {end }}[\tau]$ & $S_{0}$ \\
\hline r1 & 0.6 & 100000 & 221 & $9.25 \times 10^{-3}$ & $4.5 \times 10^{4}$ & 21.9 \\
r2 & 0.6 & 100000 & 210 & $1.08 \times 10^{-2}$ & $2.4 \times 10^{4}$ & 25.5 \\
r3 & 0.6 & 100000 & 200 & $1.25 \times 10^{-2}$ & $1.4 \times 10^{4}$ & 29.5 \\
r4 & 0.6 & 100000 & 192 & $1.41 \times 10^{-2}$ & $7.5 \times 10^{3}$ & 33.3 \\
r5 & 0.6 & 100000 & 180 & $1.71 \times 10^{-2}$ & $4.5 \times 10^{3}$ & 40.5 \\
r6 & 0.5 & 100000 & 272 & $5.00 \times 10^{-3}$ & $4.0 \times 10^{4}$ & 97.8 \\
r7 & 0.5 & 10000 & 126 & $5.00 \times 10^{-3}$ & $5.1 \times 10^{4}$ & 98.4 \\
r8 & 0.5 & 100000 & 250 & $6.40 \times 10^{-3}$ & $1.8 \times 10^{4}$ & 126 \\
r9 & 0.5 & 100000 & 240 & $7.23 \times 10^{-3}$ & $1.08 \times 10^{4}$ & 142 \\
r10 & 0.4 & 100000 & 420 & $1.35 \times 10^{-3}$ & $2.0 \times 10^{5}$ & 673 \\
r11 & 0.4 & 10000 & 195 & $1.35 \times 10^{-3}$ & $3.3 \times 10^{5}$ & 673 \\
r12 & 0.4 & 100000 & 388 & $1.71 \times 10^{-3}$ & $1.0 \times 10^{5}$ & 854 \\
r13 & 0.4 & 100000 & 330 & $2.78 \times 10^{-3}$ & $2.4 \times 10^{4}$ & $1.39 \times 10^{3}$ \\
r14 & 0.4 & 100000 & 300 & $3.70 \times 10^{-3}$ & $9.9 \times 10^{3}$ & $1.85 \times 10^{3}$ \\
r15 & 0.3 & 200000 & 957 & $2.28 \times 10^{-4}$ & $9.0 \times 10^{5}$ & $2.70 \times 10^{4}$ \\
r16 & 0.3 & 50000 & 513 & $3.70 \times 10^{-4}$ & $3.5 \times 10^{5}$ & $4.39 \times 10^{4}$ \\
r17 & 0.3 & 10000 & 250 & $6.40 \times 10^{-4}$ & $1.05 \times 10^{5}$ & $7.59 \times 10^{4}$ \\
r18 & 0.3 & 10000 & 210 & $1.08 \times 10^{-3}$ & $3.0 \times 10^{4}$ & $1.28 \times 10^{5}$ \\
r19 & 0.2 & 10000 & 700 & $2.92 \times 10^{-5}$ & $2.4 \times 10^{6}$ & $2.32 \times 10^{8}$ \\
r20 & 0.2 & 10000 & 600 & $4.63 \times 10^{-5}$ & $1.5 \times 10^{6}$ & $3.68 \times 10^{8}$ \\
\hline \hline
\end{tabular}

where $\sigma$ and $\varepsilon$ are the parameters of length and energy of the potential, which are set to be the unit length and energy, respectively, in the MD simulation of the present study. The mass unit is monomers mass. For argon, the units are given by $\varepsilon / k=119.8 \mathrm{~K}, \sigma=3.405 \AA$, and $m=6.634 \times 10^{-23} \mathrm{~g}$.

We started the simulation under the initial condition that monomers are located randomly. The computational region is a cube with a side $L$ of (100-1000) $\sigma$ having periodic boundaries. Varying the box size $L$, we set the initial number density of molecules (or the initial supersaturation ratio). In order to evaluate the nucleation rates accurately, many nuclei (at least ten or more) should be formed in the calculation time. We chose a particle number $N$ (and $L$ ) based on this criterion. To see the dependence on the box size, we included two cases in which the supersaturation ratio is almost the same but $N$ and $L$ are different (r6 and r7 at $k T=0.5 \varepsilon$ and r10 and r11 at $k T=0.4 \varepsilon$ ).

The motions of each molecule were integrated using the leap-frog scheme. The time step was set to be $0.01 \tau$, where the time unit $\tau$ ( $=\sqrt{m \sigma^{2} / \varepsilon}$ ) is $2.16 \mathrm{ps}$ in the argon system. We took NVT (constant volume and temperature) ensembles. Simulations were performed for various temperatures, i.e., $T=(0.2-0.6) \varepsilon / k$. In order to keep the temperature constant, we used a simple velocity scaling method, as described in our previous study. ${ }^{36}$ The calculation of the interaction between molecules is truncated at $5 \sigma$. This truncation length would be large enough to reproduce the vapor properties. ${ }^{54}$ We used the cell index method to search for interacting neighbors within the cutoff distance. ${ }^{55,56}$ This reduces the computation time significantly and enables the motion of molecules to be calculated for a long period of time. The values of the parameter set for each run are shown in Table I.

In order to define clusters in the numerical data, we adopt the following condition for the bounding of two
TABLE II. Thermodynamic quantities and parameters at each temperature.

\begin{tabular}{lccccccc}
\hline \hline$k T / \varepsilon$ & $P_{\text {sat }}^{\mathrm{a}}\left[\varepsilon / \sigma^{3}\right]$ & $\sigma_{s}^{\mathrm{b}}\left[\varepsilon / \sigma^{2}\right]$ & $\rho_{\mathrm{m}}^{\mathrm{c}}\left[m / \sigma^{3}\right]$ & $\mathrm{B}_{2} / \sigma^{3}$ & $\eta$ & $\xi$ & $r_{\mathrm{c}}[\sigma]$ \\
\hline 0.6 & $2.54 \times 10^{-4}$ & 1.32 & 0.882 & -13.0 & 11.6 & -6.22 & 1.41 \\
0.5 & $2.54 \times 10^{-5}$ & 1.57 & 0.921 & -18.3 & 16.1 & -9.45 & 1.46 \\
0.4 & $8.02 \times 10^{-7}$ & 1.83 & 0.958 & -28.9 & 22.8 & -13.9 & 1.52 \\
0.3 & $2.53 \times 10^{-9}$ & 2.10 & 0.993 & -58.4 & 33.9 & -20.8 & 1.60 \\
0.2 & $2.51 \times 10^{-14}$ & 2.37 & 1.03 & -232 & 56.3 & -33.9 & 1.72 \\
\hline \hline
\end{tabular}

${ }^{\mathrm{a}}$ Reference 54

${ }^{\mathrm{b}}$ Reference 57

${ }^{\mathrm{c}}$ Reference 47

monomers: ${ }^{36,51}$

$$
\frac{m}{4} v^{2}+u_{i j}(r) \leq 0
$$

where $v$ is the relative velocity between two molecules. The square of the velocity $v^{2}$ is set to be $3 \mathrm{kT} / \mathrm{m}$ as the thermodynamic average. ${ }^{36}$ This condition can be expressed in the form $r<r_{\mathrm{c}}$, where the values of the bounding distance $r_{\mathrm{c}}$ are $(1.4-1.7) \sigma$ for $T=(0.2-0.6) \varepsilon / k$. In the analysis of the numerical data of the MD simulations, a number of thermodynamic quantities in the Lennard-Jones systems, such as saturated vapor pressure, surface tension, and bulk density of the condensed phase, are needed. We assume that the clusters are first nucleated as supercooled liquid droplets. This assumption is supported by the numerical results. The empirical formulae of these thermodynamic quantities and related references are described in Appendix B. The second virial constant is also necessary in the SP model. In order to obtain the second virial constant, we numerically integrate Eq. (B3). The non-dimensional parameters $\eta$ and $\xi$ are calculated from these quantities. The values of these thermodynamic quantities and the bounding distance $r_{\mathrm{c}}$ for each temperature are shown in Table II.

\section{RESULTS}

\section{A. A typical case}

We first show the results for a typical case of MD simulations in which $T=0.3 \varepsilon / k$ and the initial supersaturation

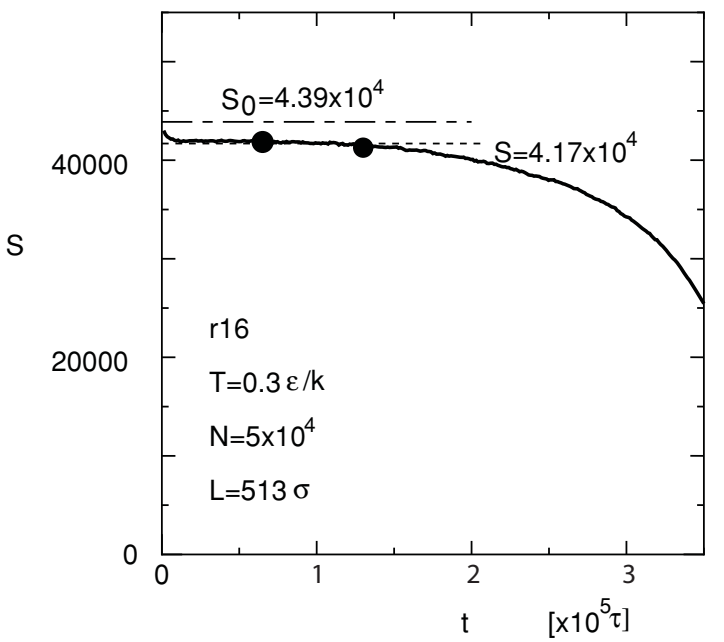

FIG. 1. Time evolution of the supersaturation ratio $S$ in a typical case with $T=0.3 \varepsilon / k$ and the initial supersaturation ratio $S_{0}=4.39 \times 10^{4}$ (r16). For the period of $t=(0.7-1.4) \times 10^{5} \tau$ (bounded with closed circles), the mean value of $S$ is $4.17 \times 10^{4}$. 
(a)

(b)

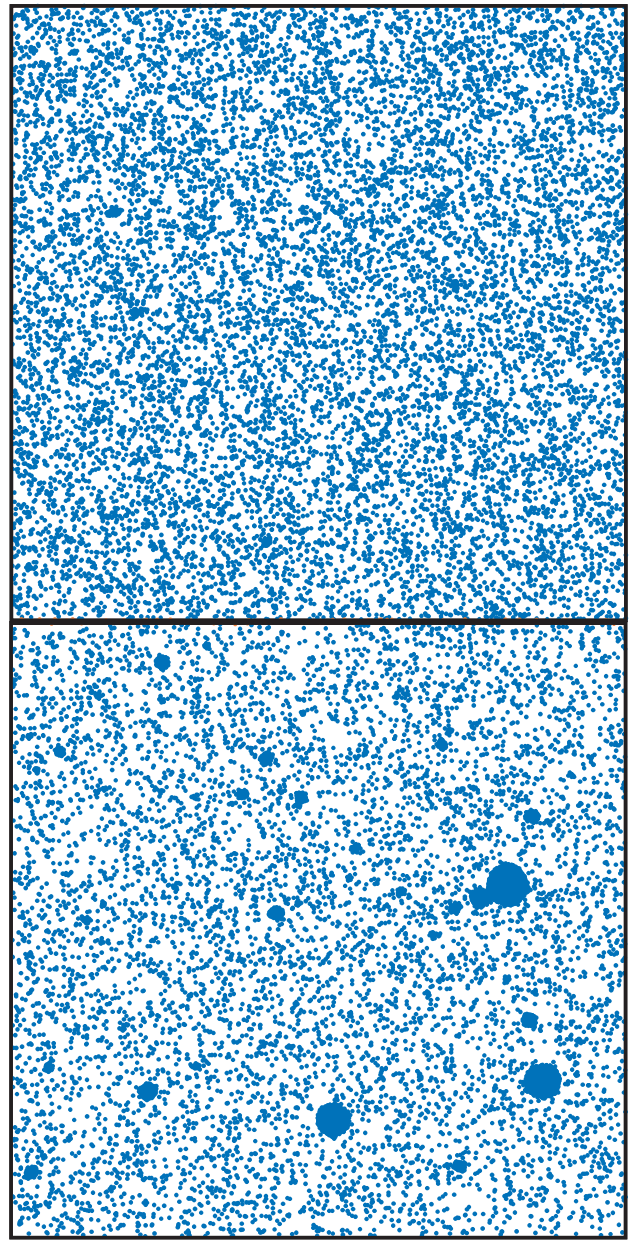

FIG. 2. Snapshots at $t=1.5 \times 10^{5} \tau$ (a) and $3.5 \times 10^{5} \tau$ (b) for the typical case of $\mathrm{r} 16$.

ratio is $S_{0}=4.39 \times 10^{4}$ (r16 in Table I). Figure 1 shows the time evolution of the supersaturation ratio $S\left(=P_{1} / P_{\text {sat }}\right)$, and Fig. 2 shows snapshots of the run at $t=1.5 \times 10^{5} \tau$ and $3.5 \times 10^{5} \tau$. In the early stage $\left(t<1.5 \times 10^{5} \tau\right)$, the supersaturation ratio remains approximately constant $(S \simeq 41700$ ). At this stage, there exist a large number of unstable clusters, and stable clusters $\left(i>i^{*}\right)$ are gradually nucleated. At the end of this nucleation stage, the nuclei are still small, as shown in Fig. 2(a). In the latter stage $\left(t>1.5 \times 10^{5} \tau\right)$, stable nuclei grow significantly, and $S$ decreases rapidly. In Fig. 2(b), the largest cluster contains 2073 molecules. We refer to this latter stage as a growth stage.

By counting the number of stable clusters in MD simulations, we can obtain the nucleation rate. Let $N\left(>i_{\text {th }}\right)$ denote the number of clusters larger than a threshold size $i_{\text {th }}$. Figure 3 shows the time evolution of $N\left(>i_{\text {th }}\right)$ for $i_{\text {th }}=10$ and 20 . The threshold $i_{\text {th }}$ should be larger than the critical size $i^{*}$. In this case, the critical size $i^{*}$ is estimated to be 5 in the SP model. In this figure, the number of stable nuclei $N\left(>i_{\text {th }}\right)$ increases almost linearly in the period of $t=(0.7-1.4) \times 10^{5} \tau$. Based on the slope, the nucleation rate $J\left(=\dot{N}\left(>i_{\text {th }}\right) / L^{3}\right)$ is evaluated to be $1.3 \times 10^{-12} \sigma^{-3} \tau^{-1}$ (or $J=1.6 \times 10^{22} \mathrm{~cm}^{-3} \mathrm{~s}^{-1}$ for argon).

After the nucleation stage, $N\left(>i_{\text {th }}\right)$ is approximately constant for a time, but then increases again at $t>2.5$

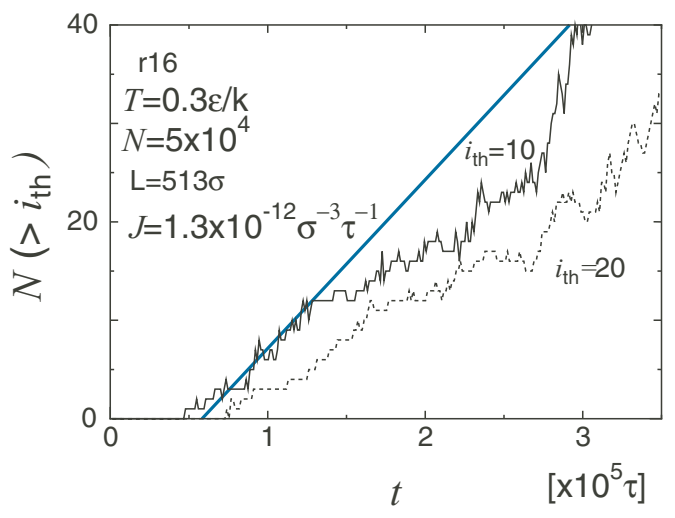

FIG. 3. Time evolution of $N\left(>i_{\text {th }}\right)$, which is the number of clusters larger than the threshold size, $i_{\text {th }}$ for the typical case of r16. At the solid line (dotted line), $i_{\text {th }}$ is set to be $10(20)$. Based on the slope of $N\left(>i_{\text {th }}\right)$ for $t=(0.7-1.4) \times 10^{5} \tau$ (solid blue line), the nucleation rate is evaluated to be $J=1.3 \times 10^{-12} \sigma^{-3} \tau^{-1}$ (or $J=1.6 \times 10^{22} \mathrm{~cm}^{-3} \mathrm{~s}^{-1}$ ).

$\times 10^{5} \tau$. This second nucleation in the growth stage is explained as follows. During the growth stage, the growing nuclei are heated by latent heat deposition, which makes them hotter than the vapor (or unstable small clusters). On the other hand, the entire system is cooled by a thermostat in the simulation so that the average temperature is maintained constant. This artificial decreasing of the temperature of the vapor efficiently increases the supersaturation ratio, which causes the second nucleation. Hence, we do not focus on the artificial second nucleation.

During the period of $t=(0.7-1.4) \times 10^{5} \tau$, in which the nucleation proceeds, the averaged value of $S$ is 4.17 $\times 10^{4}$. Using this value, we can calculate $J$-values based on the modified-CNT or the SP model assuming $\alpha=1$. In the modified-CNT, the nucleation rate is evaluated to be $1.0 \times$ $10^{-19} \sigma^{-3} \tau^{-1}$, whereas $J=3.8 \times 10^{-11} \sigma^{-3} \tau^{-1}$ in the SP model. The modified-CNT underestimates the nucleation rate obtained by the MD simulation by a factor of $10^{-7}$, while the SP model overestimates it by a factor of 10 . The SP model enables much more accurate prediction than the modified-CNT. The validity of the assumption that $\alpha=1$ will be discussed later.

Figure 4 shows size distributions of the clusters for the typical case. The size distribution is averaged over the time period from $0.7 \times 10^{5} \tau$ to $1.4 \times 10^{5} \tau$ in the nucleation stage. We also plotted the size distributions calculated using the SP model and the modified-CNT, respectively. As shown in Fig. 4, the sizes of the critical cluster in the SP model and the modified-CNT are estimated to be 5 and 10, respectively. The SP model reproduces well with the size distribution of the MD simulations within a factor of 10 , whereas the prediction by the modified-CNT is much smaller than that by the MD simulation by a factor of $10^{8}$ around the critical clusters. These deviations in the size distributions are almost equivalent to the deviations in the nucleation rates.

\section{B. Nucleation rate}

Including the above typical case, we performed 20 runs of MD simulation for various values of temperature and 


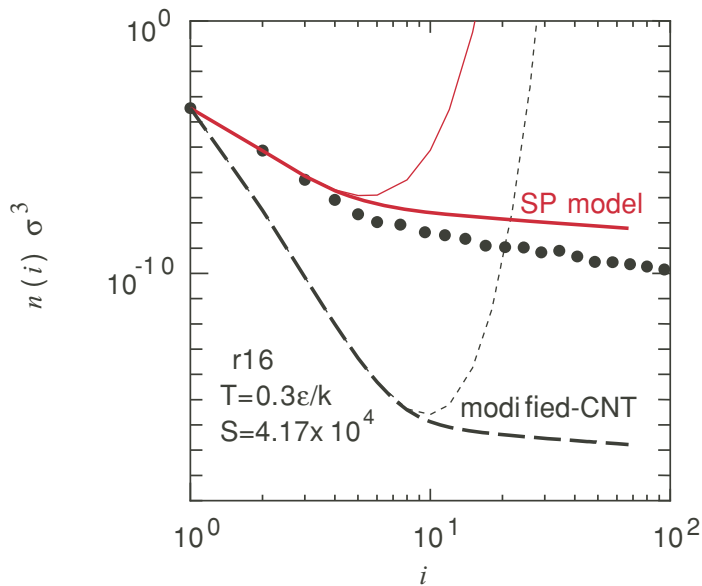

FIG. 4. Size distribution of clusters $n(i)$ obtained by the MD simulations is indicated by closed circles for the typical case of $\mathrm{r} 16$. The size distribution is averaged over the time of $(0.7-1.4) \times 10^{5} \tau$. The size distributions obtained by the SP model and the modified-CNT are plotted by solid and dashed lines, respectively. For a reference, the equilibrium distributions $n_{\mathrm{e}}(i)$ obtained by the SP model and by the modified-CNT are plotted by thin solid and dotted lines, respectively.

initial supersaturation ratio, as listed in Table I. Here, we show the overall results for the nucleation rate and compare these results with the theoretical models.

As in the typical case, the nucleation rate is evaluated in each run by counting the number of nuclei $N\left(>i_{\text {th }}\right)$. Figure 5 shows the time evolution of $N\left(>i_{\text {th }}\right)$ for three other cases. Since a large number of nuclei are formed, the nucleation rates are evaluated at higher accuracy in these cases. In the latter two cases, the nucleation rate is enhanced in the growth stage, which corresponds to the second nucleation mentioned in Subsection IV A.

In Fig. 6, the nucleation rates obtained from all of the MD simulations are plotted by open circles. (The values of the obtained nucleation rates are also listed in Table III.) Wedekind et al $^{43}$ performed similar MD simulations in a lowtemperature region for a system consisting of 343 molecules. We also plotted their results at $T=0.5 \varepsilon / k$ by filled triangles. Their results agree well with the results of the present study.

The numerical results are compared with the theoretical estimations in Fig. 6. The theoretical values are also listed in Table III. Note that the nucleation rate predicted by the SP model agrees well with the MD simulations, whereas the estimations using the modified-CNT are by many orders of magnitudes smaller than those of the MD simulations. We also confirmed that the original classical theory yields smaller estimations than the modified-CNT. It should be noted that there are some uncertainties in the saturation vapor pressure and the surface tension, in particular, at the low temperature (see also Appendix B). The uncertainty of a factor of 2 in the vapor pressure (or $10 \%$ in the surface tension) causes the uncertainty in the nucleation rate about a factor of 2 for $T=0.2 \varepsilon / k$. Table III lists also the sizes of the critical cluster obtained by the SP model $i_{\text {SP }}^{*}$. For all cases, the sizes of the critical clusters are smaller than 20 . The size of the critical cluster can be obtained from the slope of the nucleation rates of MD simulations in Fig. 6 , since $i^{*}$ is approximately given by $\partial \ln J / \partial \ln S{ }^{37,43}$
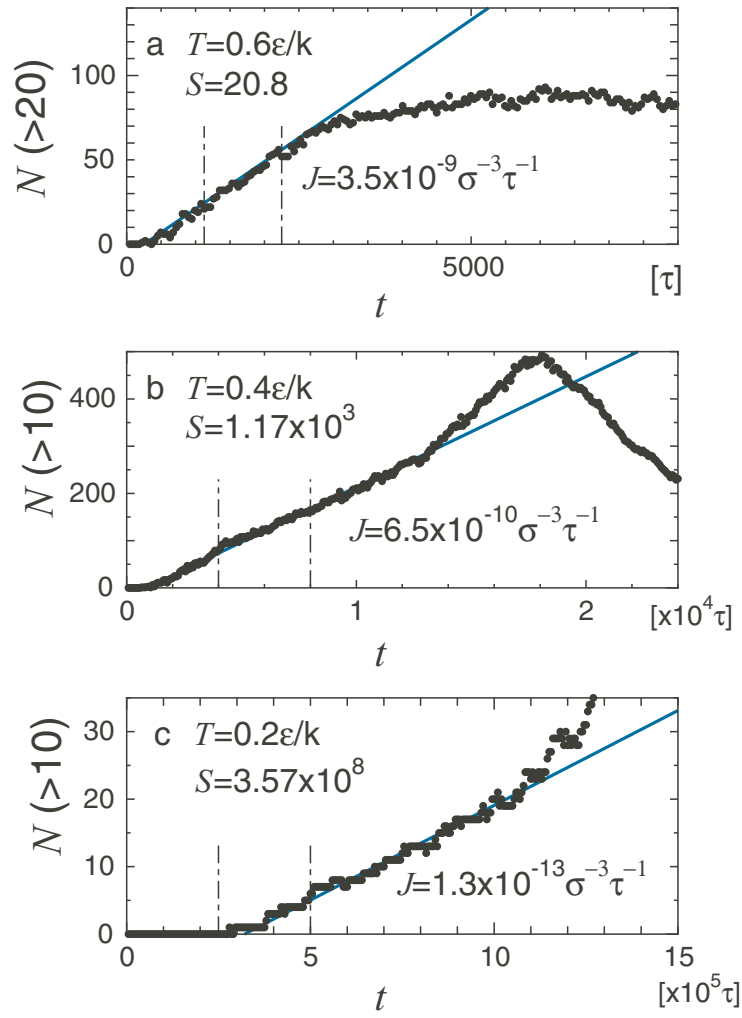

FIG. 5. Time evolution of $N\left(>i_{\text {th }}\right)$ for three other cases. For the case of r3 $\left(k T=0.6 \varepsilon, S_{0}=29.5\right)$ in (a), we set $i_{\text {th }}=20$. For the cases of r13 $\left(k T=0.4 \varepsilon, S_{0}=1.39 \times 10^{3}\right)$ in $(\mathrm{b})$ and $\mathrm{r} 20\left(k T=0.2 \varepsilon, S_{0}=3.68 \times 10^{8}\right)$ in (c), we set $i_{\text {th }}=10$. Based on the slopes of $N\left(>i_{\text {th }}\right)$ in the period shown by the dashed-dotted lines in each panel (solid lines), the nucleation rates in (a) through (c) are evaluated to be $3.5 \times 10^{-9}, 6.5 \times 10^{-10}$, and $1.3 \times 10^{-13} \sigma^{-3} \tau^{-1}$, respectively. The values of the supersaturation ratio averaged over the period shown by the dashed-dotted lines in each panel are $20.8,1.17 \times 10^{3}$, and $3.57 \times 10^{8}$, respectively.

As shown in Fig. 6, the slopes of MD simulations are in agreement with those of the SP model. This agreement suggests that the size of the critical cluster in the MD simulations is consistent with the SP model.

In order to clarify the deviations from the numerical results, the ratios of nucleation rates obtained by the SP model $J_{\mathrm{SP}}$ (or modified-CNT $J_{\mathrm{MCNT}}$ ) to those obtained by MD simulations $J_{\mathrm{MD}}$ are plotted in Fig. 7. The overestimations of $J$ of the SP model from the numerical results are around a factor of 10 in all cases. A possible reason of the deviation of the SP model will be discussed later herein. The modified-CNT underestimates the nucleation rate by a factor of $10^{-3}\left(10^{-10}\right)$ at $T=0.6 \varepsilon / k(0.2 \varepsilon / k)$. At the lower temperature, the error of the modified-CNT is significant. This figure clearly shows that the correction in the SP model works very well in the entire range of temperature considered herein.

\section{Cluster size distributions}

In a typical case (i.e., in Fig. 4), the size distribution of clusters in the MD simulation is found to be well reproduced by the SP model, whereas the modified-CNT significantly underestimates the number of clusters. Figure 8 shows the size distributions $n(i)$ obtained in 10 more MD simulation runs 
TABLE III. Summary of the results of the MD simulations. $T$ : temperature, $S$ : averaged supersaturation ratio in the nucleation stage, $J_{\mathrm{MD}}$ : nucleation rates obtained by the MD simulations, $J_{\mathrm{SP}}$ : nucleation rates by the SP model, $J_{\mathrm{MCNT}}$ : nucleation rates by the modified-CNT,$i_{\mathrm{SP}}^{*}$ : critical cluster size evaluated by the SP model, and $\alpha$ : sticking probability.

\begin{tabular}{|c|c|c|c|c|c|c|c|}
\hline Run\# & $T[\varepsilon / k]$ & $S$ & $J_{\mathrm{MD}}\left[\sigma^{-3} \tau^{-1}\right]^{\mathrm{a}}$ & $J_{\mathrm{SP}}\left[\sigma^{-3} \tau^{-1}\right]$ & $J_{\mathrm{MCNT}}\left[\sigma^{-3} \tau^{-1}\right]$ & $i_{\mathrm{SP}}^{*}$ & $\alpha$ \\
\hline $\mathrm{r} 1$ & 0.6 & 16.9 & $0.5 \times 10^{-10}$ & $1.5 \times 10^{-9}$ & $7.6 \times 10^{-14}$ & 15 & 0.18 \\
\hline $\mathrm{r} 2$ & 0.6 & 18.5 & $4.0 \times 10^{-10}$ & $5.8 \times 10^{-9}$ & $5.2 \times 10^{-13}$ & 13 & 0.20 \\
\hline $\mathrm{r} 3$ & 0.6 & 20.8 & $3.5 \times 10^{-9}$ & $2.7 \times 10^{-8}$ & $4.6 \times 10^{-12}$ & 11 & 0.29 \\
\hline $\mathrm{r} 4$ & 0.6 & 22.2 & $1.5 \times 10^{-8}$ & $5.6 \times 10^{-8}$ & $1.3 \times 10^{-11}$ & 11 & 0.38 \\
\hline $\mathrm{r} 5$ & 0.6 & 24.6 & $1.0 \times 10^{-7}$ & $1.7 \times 10^{-7}$ & $7.0 \times 10^{-11}$ & 9 & 0.65 \\
\hline r6 & 0.5 & 80.0 & $0.7 \times 10^{-10}$ & $4.0 \times 10^{-9}$ & $2.4 \times 10^{-14}$ & 9 & 0.20 \\
\hline $\mathrm{r} 7$ & 0.5 & 80.2 & $1.0 \times 10^{-10}$ & $4.2 \times 10^{-9}$ & $2.5 \times 10^{-14}$ & 9 & 0.20 \\
\hline r8 & 0.5 & 96.8 & $2.0 \times 10^{-9}$ & $2.6 \times 10^{-8}$ & $4.3 \times 10^{-13}$ & 8 & 0.39 \\
\hline r9 & 0.5 & 106 & $6.0 \times 10^{-9}$ & $5.3 \times 10^{-8}$ & $1.3 \times 10^{-12}$ & 8 & 0.43 \\
\hline $\mathrm{r} 10$ & 0.4 & 616 & $4.0 \times 10^{-12}$ & $1.1 \times 10^{-10}$ & $7.7 \times 10^{-18}$ & 8 & 0.20 \\
\hline $\mathrm{r} 11$ & 0.4 & 619 & $8.0 \times 10^{-12}$ & $1.2 \times 10^{-10}$ & $8.3 \times 10^{-18}$ & 8 & 0.21 \\
\hline $\mathrm{r} 12$ & 0.4 & 769 & $1.5 \times 10^{-11}$ & $7.5 \times 10^{-10}$ & $1.6 \times 10^{-16}$ & 7 & 0.25 \\
\hline r13 & 0.4 & $1.17 \times 10^{3}$ & $6.5 \times 10^{-10}$ & $1.5 \times 10^{-8}$ & $2.2 \times 10^{-14}$ & 6 & 0.42 \\
\hline r14 & 0.4 & $1.50 \times 10^{3}$ & $6.0 \times 10^{-9}$ & $6.7 \times 10^{-8}$ & $2.8 \times 10^{-13}$ & 5 & 0.65 \\
\hline $\mathrm{r} 15$ & 0.3 & $2.59 \times 10^{4}$ & $0.3 \times 10^{-13}$ & $1.4 \times 10^{-12}$ & $4.6 \times 10^{-22}$ & 6 & 0.20 \\
\hline r16 & 0.3 & $4.17 \times 10^{4}$ & $1.3 \times 10^{-12}$ & $3.8 \times 10^{-11}$ & $1.0 \times 10^{-19}$ & 5 & 0.25 \\
\hline $\mathrm{r} 17$ & 0.3 & $6.50 \times 10^{4}$ & $3.5 \times 10^{-11}$ & $5.3 \times 10^{-10}$ & $8.9 \times 10^{-18}$ & 5 & 0.40 \\
\hline $\mathrm{r} 18$ & 0.3 & $1.11 \times 10^{5}$ & $5.5 \times 10^{-10}$ & $8.6 \times 10^{-9}$ & $1.1 \times 10^{-15}$ & 4 & 0.50 \\
\hline r19 & 0.2 & $2.23 \times 10^{8}$ & $2.8 \times 10^{-14}$ & $2.5 \times 10^{-13}$ & $9.8 \times 10^{-25}$ & 4 & 0.43 \\
\hline $\mathrm{r} 20$ & 0.2 & $3.57 \times 10^{8}$ & $1.3 \times 10^{-13}$ & $2.3 \times 10^{-12}$ & $4.5 \times 10^{-23}$ & 4 & 0.46 \\
\hline
\end{tabular}

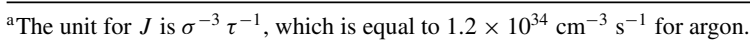

for various temperatures and initial supersaturation ratios. The left-hand column shows high- $S$ cases, and the right-hand column corresponds to low- $S$ cases for the same temperatures. The overall tendency is similar to the typical case. In all cases, the SP model predicts the size distributions very well, although overestimates by a factor of 10 occur at $T=0.4$ and $0.5 \varepsilon / k$. The prediction by the modified-CNT yields significant underestimates (by factors of $10^{-11}-10^{-3}$ ). These results

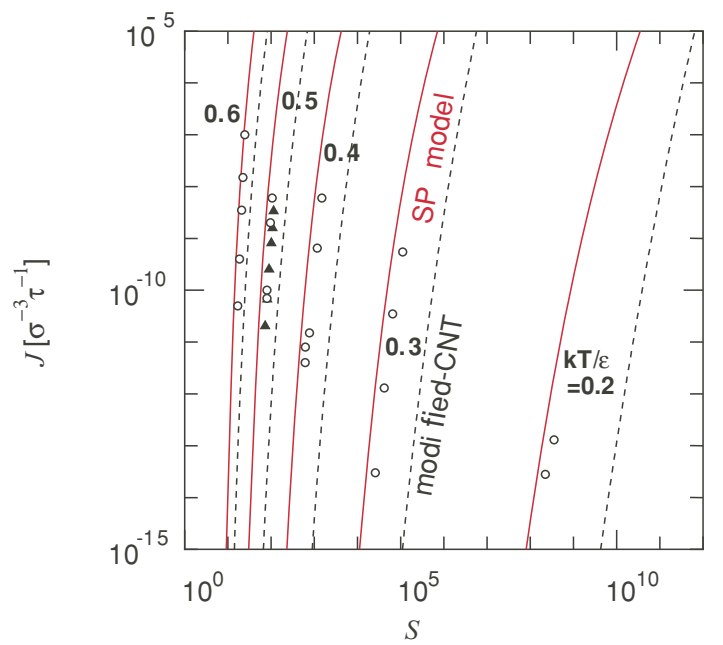

FIG. 6. Nucleation rates obtained from the present MD simulations plotted by open circles. The results obtained by Wedekind et al. (also see Ref. 43) for $T=0.5 \varepsilon / k$ are plotted by filled triangles. The curves indicate the nucleation rates calculated by the SP model (solid lines) and the modified-CNT (dashed lines). The nucleation rate predicted by the SP model agrees well with the MD simulations, whereas the estimations obtained by the modified-CNT are much smaller than the results obtained by the MD simulations. of the size distributions are consistent with the nucleation rate.

\section{Cluster integrals and the formation free energy}

As described in Sec. II, the equilibrium size distribution $n_{\mathrm{e}}(i)$ is determined by the cluster integrals $b_{i}$ given by Eq. (A3), and $n_{\mathrm{e}}(i)$ agrees well with the distributions $n(i)$ obtained by the MD simulations for $i \leq i^{*}$ (see Fig. 4). Thus, using the cluster size distributions obtained by the MD simulations, we can estimate the values of the cluster integrals for each cluster size and each temperature. Figure 9(a) shows

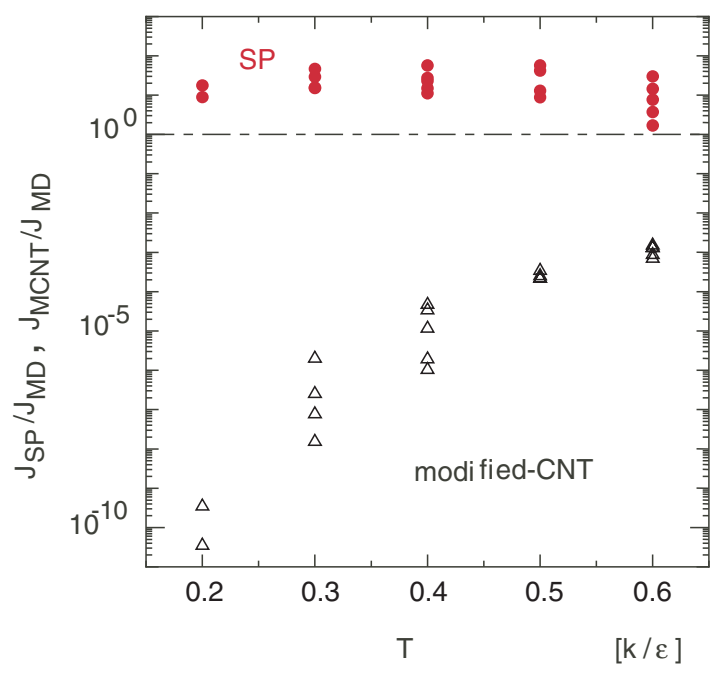

FIG. 7. Ratios of nucleation rates as a function of temperature. The ratio $J_{\mathrm{SP}} / J_{\mathrm{MD}}\left(J_{\mathrm{MCNT}} / J_{\mathrm{MD}}\right)$ is plotted by solid circles (open triangles). 


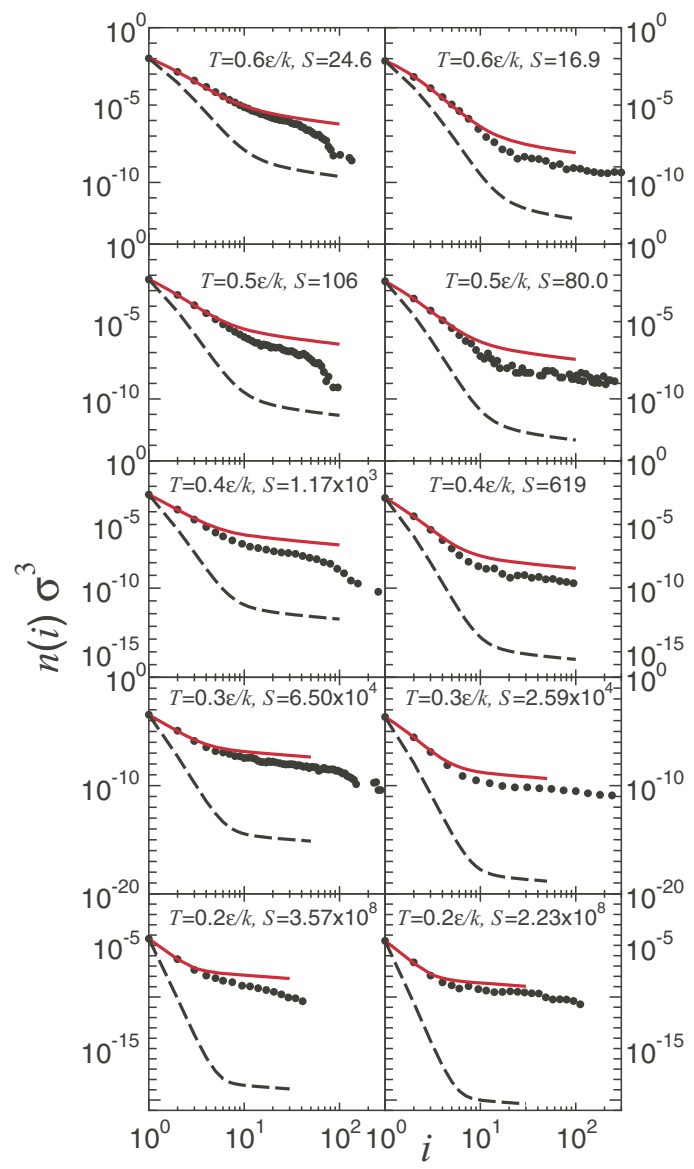

FIG. 8. As in Fig. 4 but for 10 more MD simulation runs for temperatures of $T=(0.2-0.6) \varepsilon / k$ and initial supersaturation ratios of $S_{0} \simeq 10-10^{8}$.

the cluster integrals $b_{i}$ obtained in this manner from all MD simulation runs. Only small clusters with $i \leq i_{\mathrm{SP}}^{*}$ are plotted, where $i_{\text {SP }}^{*}$ is the critical cluster size evaluated by the SP model. Predictions by theoretical models are plotted by solid lines (SP model) and dashed lines (modified-CNT). Again, the SP model exhibits much better agreement with the numerical results than the modified-CNT. Note that at each temperature, the cluster integrals, $b_{i, \mathrm{MD}}$, obtained from the MD simulations are approximately independent of the supersaturation ratio. This is consistent with the fact that each integral $b_{i}$ depends only on temperature. In Fig. 9(b), we plot the ratios of $b_{i}$ to the prediction obtained by the modified-CNT, $b_{i, \mathrm{MCNT}}$. From Eq. (16), the relation between $b_{i}$ and $\Delta G_{i}$ is given by

$$
\left(\Delta G_{i}-\Delta G_{i, \mathrm{MCNT}}\right) / k T=-\ln \left(b_{i} / b_{i, \mathrm{MCNT}}\right) .
$$

Hence, Fig. 9(b) also shows the deviation of the formation free energy $\Delta G_{i}$ from the prediction by the modified-CNT. In the SP model, the energy difference is given by $\Delta G_{i, \mathrm{SP}}-$ $\Delta G_{i, \mathrm{MCNT}}=\xi\left(i^{1 / 3}-1\right)$. Figure $9(\mathrm{~b})$ indicates that this correction by the SP model works well, at least for clusters with masses $i \lesssim 10$. On the other hand, according to the scaling law proposed by McGraw and Laaksonen, ${ }^{38,39}$ the deviation of the formation energy from the CNT prediction (or from the modified-CNT prediction) is given by a temperaturedependent constant and is independent of the cluster size $i$.
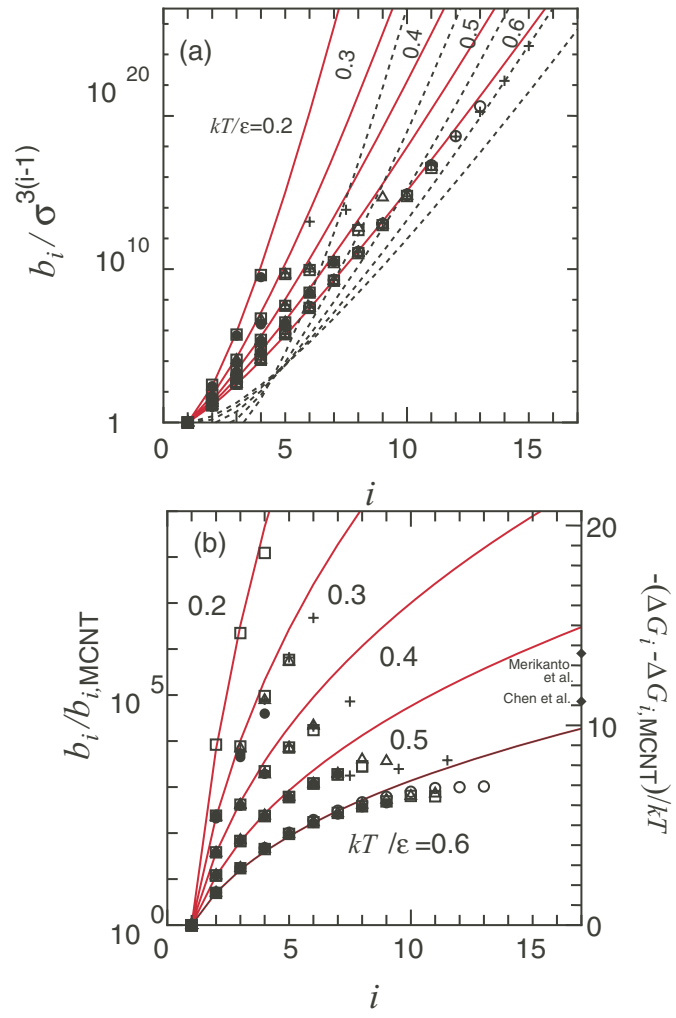

FIG. 9. (a) Cluster integrals $b_{i}$ obtained from all MD simulations. Different symbols indicate $b_{i}$ obtained from the MD simulations starting from different supersaturation ratios $S_{0}$. The values obtained by the SP model (solid lines) and by the modified-CNT (dotted lines) are also shown. (b) The ratio of $b_{i}$ to the prediction by the modified-CNT, $b_{i, \mathrm{MCNT}}$, is plotted. Various symbols represent $b_{i, \mathrm{MD}}$, and the solid lines indicate $b_{i, \mathrm{SP}}$, respectively. The right-hand axis corresponds to the deviation of the formation free energy $\Delta G_{i}$ obtained from the modified-CNT. In addition, the values suggested by Merikanto et al. (also see Ref. 45) and Chen et al. (also see Ref. 46) are plotted by filled diamonds.

At relatively large clusters (with $i \gtrsim 30$ for L-J molecules), the scaling law has been confirmed by several authors by means of Monte-Carlo and MD simulations. ${ }^{22,31,42,45,48,49}$ For example, the deviation from the modified-CNT prediction $\Delta G_{i}-\Delta G_{i, \mathrm{MCNT}}$ is estimated to be $-13.6 k T$ by Merikanto et $a l^{45}$ and $-11.2 k T$ by Chen et $a l .{ }^{46}$ for large clusters. These values are plotted in Fig. 9(b). The results of the present study suggest that the formation energy of clusters smaller than $\sim 15$ is well described by the SP model, in contrast to the scaling law proposed by McGraw and Laaksonen.

\section{E. Cluster growth and sticking probability}

As shown in Sec. II, the nucleation rate is obtained from the equilibrium number density $n_{e}(i)$ and the accretion rate $R^{+}(i)$. Although the accretion rate is proportional to the sticking probability $\alpha$, the sticking probability was assumed to be unity in most theoretical models of nucleation. Using the growth rate of stable clusters obtained in the present MD simulation, we can evaluate the sticking probability in the following manner. 

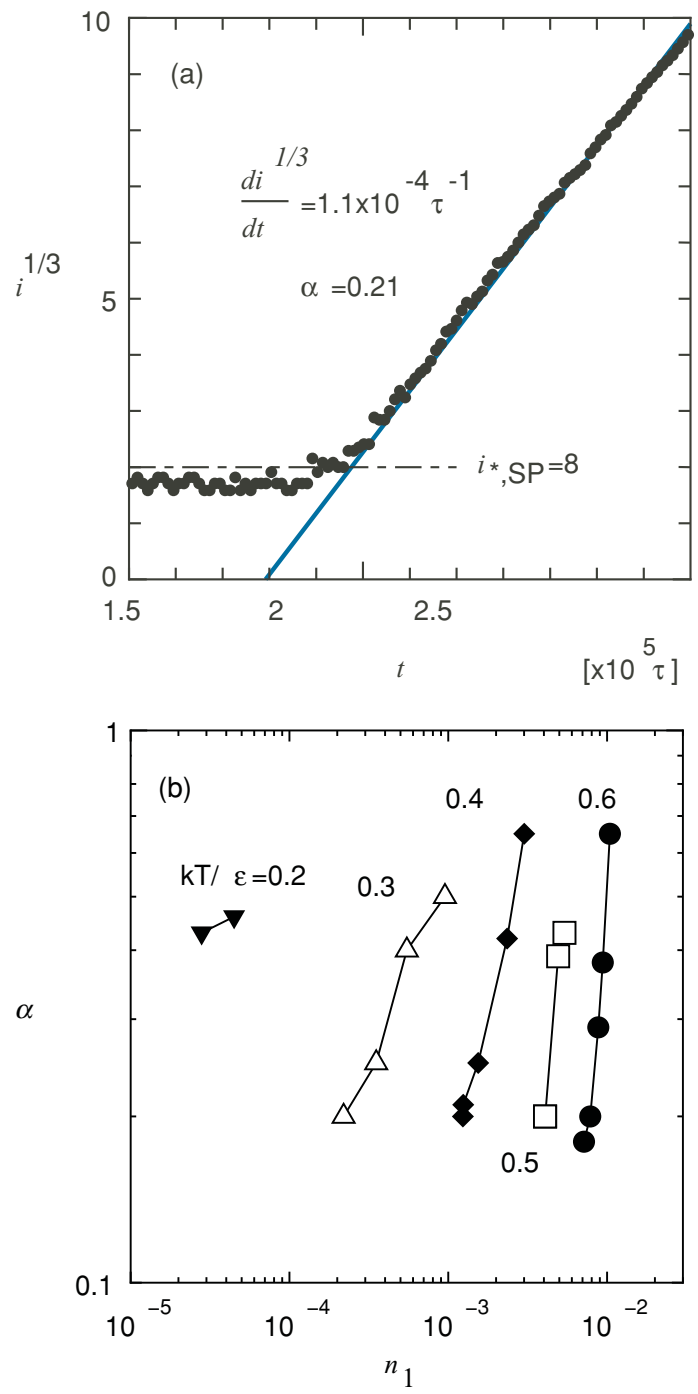

FIG. 10. (a) Time evolution of the size of the largest cluster for the case of r11. Note that for $t \lesssim 2.0 \times 10^{5} \tau$ the cluster size keeps a constant value which corresponds to the critical cluster size predicted by the SP model, $i_{\mathrm{SP}}^{*}=8$. Based on the slope (solid line) of the cluster growth, the sticking probability is evaluated to be 0.21 . (b) Sticking probability obtained from all MD simulations.

In a supersaturated state, evaporation is negligible for stable clusters larger than the critical size $i^{*}$, and the growth rate of stable clusters, $d i / d t$, is given as

$$
\frac{d i}{d t}=R^{+}(i)=\alpha n(1) v_{\text {th }}\left(4 \pi r_{0}^{2} i^{2 / 3}\right),
$$

where we used Eq. (2). Solving this equation for $\alpha$, we obtain

$$
\alpha=\frac{3}{4 \pi r_{0}^{2} v_{\mathrm{th}} n(1)} \frac{d i^{1 / 3}}{d t} .
$$

The time derivative of $i^{1 / 3}$ is evaluated from the MD simulations. For example, Fig. 10(a) shows the growth of the largest cluster for the case of $\mathrm{r} 11$, where $T=0.4 \varepsilon / k$ and $S_{0}=673$. Based on the slope, $d i^{1 / 3} / d t$ is estimated to be $1.1 \times 10^{-4} / \tau$ and the sticking probability is obtained as 0.21 . In this estimation, the value of $n(1)$ is calculated using the average supersaturation ratio $S$. The linearity of $i^{1 / 3}$ in Fig. 10(a) indicates that the sticking probability is independent of the cluster size.

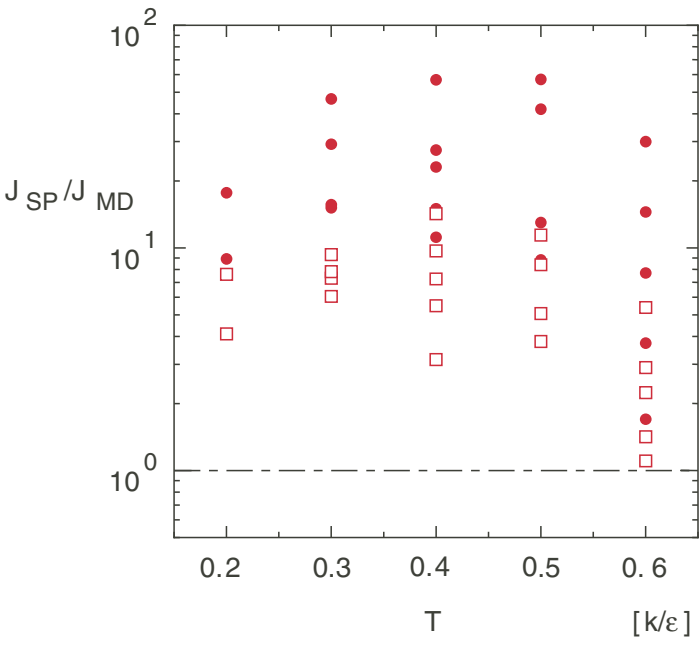

FIG. 11. The ratios between the nucleation rates $J_{\mathrm{SP}} / J_{\mathrm{MD}}$. For the points indicated by the filled circles, $\alpha$ is assumed unity, whereas the values of $\alpha$ obtained by the MD simulations are used for the points indicated by the open squares.

Using this method, we obtained the sticking probability from all runs of the MD simulations. The results are shown in Fig. 10(b) as a function of the averaged supersaturation ratio $S$. The values of $\alpha$ are also listed in Table III. The sticking probability increases with the supersaturation ratio $S$ for each of temperatures. The clusters growing in supersaturated vapor are warmer than their surroundings. The warming effect is more significant in the high- $S$ cases. If this effect reduces their growth rate (and $\alpha$ ), $\alpha$ decreases with increasing $S$. However, the $S$-dependence of $\alpha$ is opposite. With increasing $S$, the cluster surface becomes rougher because of the rapid deposition of molecules. This roughness of the surface may explain the $S$-dependence of $\alpha$. At the highest- $S$ run for each temperature, the sticking probability is larger than 0.6. The $S$-dependence is stronger at higher temperatures. Although in all runs in the present study, $\alpha>0.1$, the sticking probability is expected to be much smaller than 0.1 in cases of supersaturation ratios lower than in the present cases. In such low- $S$ cases, the assumption of $\alpha=1$ would be invalid for evaluating the nucleation rate. We refine the SP model by taking the values of $\alpha$ obtained in the MD simulations into account.

The ratios of the nucleation rates between the SP model and the MD simulations are plotted in Fig. 11, in which we consider two cases: one is the case in which $\alpha=1$ and the other is the case in which $\alpha$ is set to a value obtained through the MD simulations. By taking into account the realistic values of the sticking probability, we found that all of the deviations from the MD simulation decrease. With this refinement, the overestimations in the SP model are within a factor of 20 for all cases.

\section{F. Crystallization of clusters}

In all of the MD simulation runs, we found that the clusters first nucleated as liquid droplets. This is because of the small size of the nuclei, which is also supported by recently reported results of MC simulations ${ }^{46}$ Furthermore, during the rapid growth of the nuclei the latent heat deposition increases 
(a)

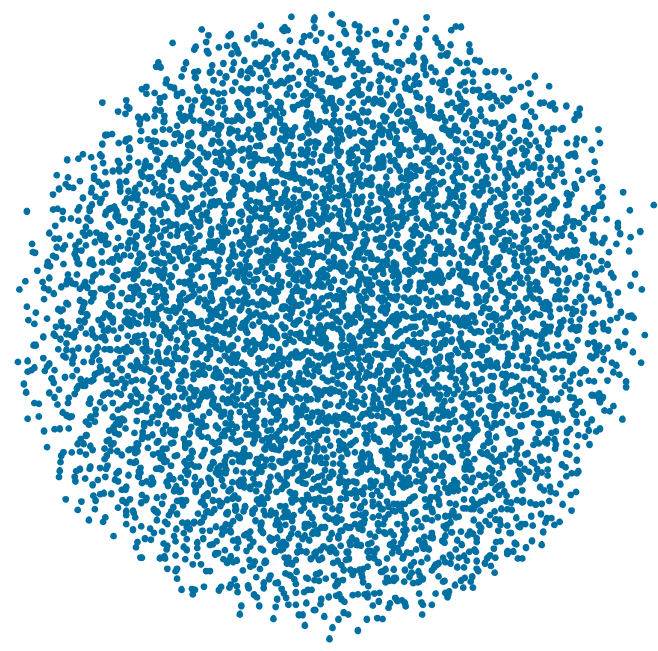

(b)

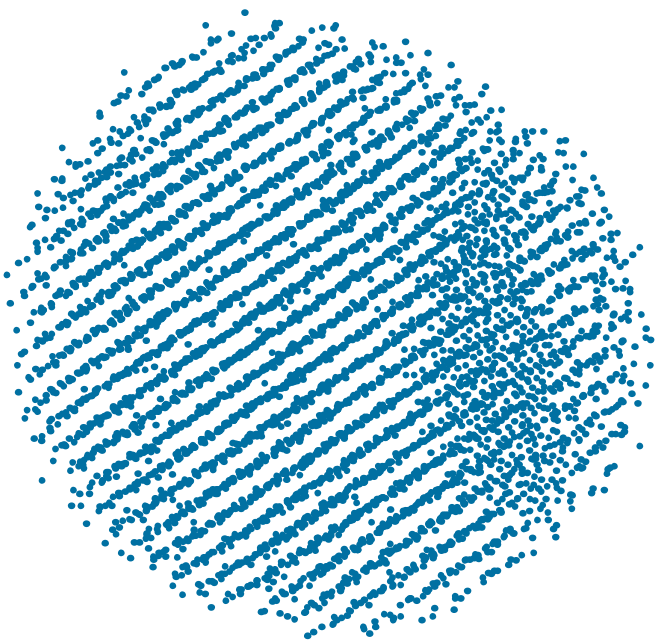

FIG. 12. Snapshots of the largest cluster at $t=3.069 \times 10^{5} \tau$ (a) and $3.080 \times 10^{5} \tau$ (b) for $T=0.4 \varepsilon / k$ and $S_{0}=673(\mathrm{r} 11)$.

the temperature of the nuclei, which also prevents their crystallization. For cases in which $T \leq 0.4 \varepsilon / k$, we observed crystallization events of supercooled liquid nuclei within the computation time. Figure 12 shows snapshots of the largest cluster at $t=3.069 \times 10^{5} \tau$ and $3.080 \times 10^{5} \tau$ for $T=0.4 \varepsilon / k$ and $S_{0}=673$ (r11). The internal structure of the cluster changes drastically within this short term of $\sim 10^{3} \tau$. Although the molecular arrangement in the cluster is random in snapshot (a), a clear lattice pattern is observed in snapshot (b). In this run, the cluster is nucleated at $t \simeq 2 \times 10^{5} \tau$, which is long before crystallization occurs. Crystallization occurs when the growth of the clusters slows due to the decrease in the number of vapor molecules. Crystallization is also confirmed by calculating the pair correlation function of the molecular positions in the cluster. The pair correlation function $g_{2}(r)$ of an $i$-mer is defined by

$$
g_{2}(r)=\frac{\left\langle N_{i}(r)\right\rangle}{4 \pi r^{2} n_{\mathrm{c}} d r},
$$

where $\left\langle N_{i}(r)\right\rangle$ is the mean number of molecules that are placed at a distance between $r$ and $r+d r$ from a reference molecule, and $n_{\mathrm{c}}=\rho_{\mathrm{c}} / m$ is the mean molecular number den-

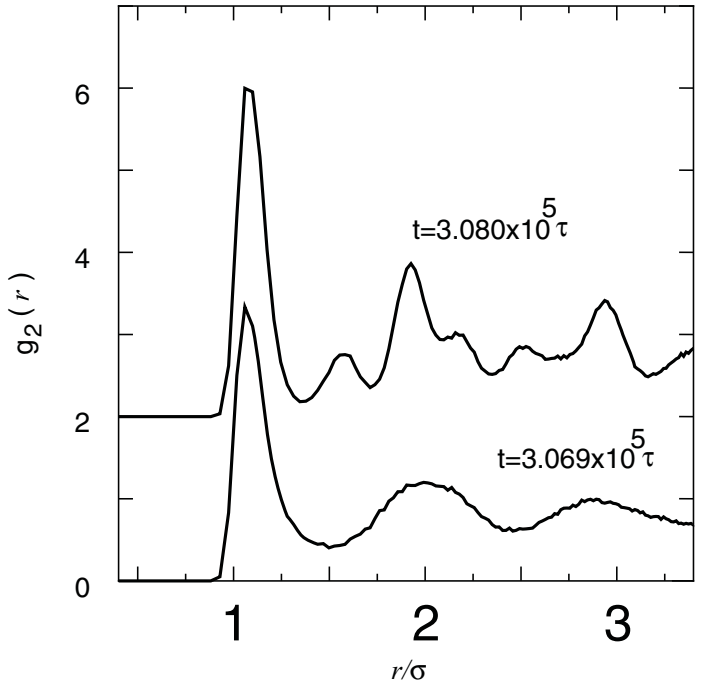

FIG. 13. Pair correlation functions in the largest cluster at $t=3.069$ $\times 10^{5} \tau$ and $3.080 \times 10^{5} \tau$ for $T=0.4 \varepsilon / k$ and $S_{0}=673(\mathrm{r} 11)$. The curve for $t=3.080 \times 10^{5} \tau$ is offset upward.

sity in the cluster where $\rho_{\mathrm{c}}$ is the mean density. We measure $n_{\mathrm{c}}$ (or $\rho_{\mathrm{c}}$ ), using the gyration radius of an $i$-mer, which is defined as

$$
r_{\mathrm{g}}^{2}=\frac{1}{i} \sum_{j=1}^{i}\left(\mathbf{r}_{j}-\mathbf{r}_{\mathrm{G}}\right)^{2}
$$

where $i$ is the number of molecules in the cluster and $r_{\mathrm{G}}$ is the center of the gravity of an $i$-mer. If we consider a sphere of constant density, the radius of the sphere is given by $\sqrt{5 / 3} r_{\mathrm{g}}$. The volume of the $i$-mer equals $(4 \pi / 3)\left(\sqrt{5 / 3} r_{\mathrm{g}}\right)^{3}$ approximately and the mean molecular number density is given as

$$
n_{\mathrm{c}}=\frac{i}{\frac{4 \pi}{3}\left(\sqrt{\frac{5}{3}} r_{\mathrm{g}}\right)^{3}} .
$$

In Fig. 13, we plot the pair correlation functions $g_{2}(r)$ of the largest cluster at $t=3.069 \times 10^{5} \tau$ and $3.080 \times 10^{5} \tau$. At $t=3.069 \times 10^{5} \tau, g_{2}(r)$ does not have peaks that are characteristic of the crystal, whereas such peaks appear at $t=3.080 \times 10^{5} \tau$. In Fig. 14, we show the time evolution of the mean density $\rho_{\mathrm{c}}$ of the cluster. A jump in the density from 0.93 to 0.97 appears in the L-J unit at $t=3.08 \times 10^{5} \tau$. This jump is due to the crystallization of the cluster. Similar crystallizations of the clusters are observed in all cases of $T \leq 0.4 \varepsilon / k$. In the higher-temperature cases, on the other hand, no crystallization is observed within the computation time of the simulations.

\section{SUMMARY AND FUTURE RESEARCH}

We performed MD simulations of nucleation from a vapor to a solid phase of L-J type molecules for a wide range of temperatures and initial supersaturation ratios. In the present study, we focused on the temperature range below the triple point, whereas higher-temperature cases of $T \gtrsim 0.7 \varepsilon / k$ were examined in our previous study. ${ }^{36}$ The results of the present study are summarized as follows. 


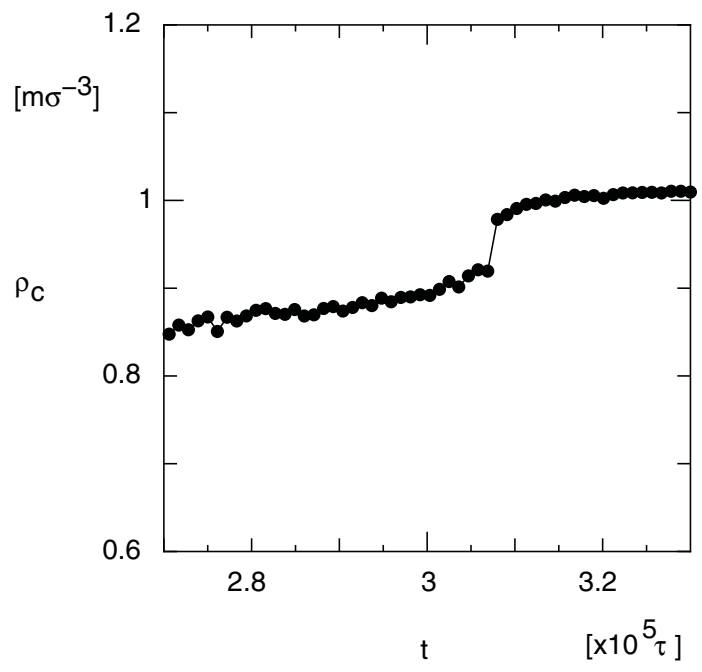

FIG. 14. Time evolution of mean density in the largest cluster for the case in which $T=0.4 \varepsilon / k$ and $S_{0}=673$ (r11). A density jump is observed at the crystallization event.

1. The nucleation rates and cluster size distributions obtained from the MD simulations are compared with the predictions by the theoretical models (see Figs. 6-8 and Table III). We found that the CNT extremely underestimates the nucleation rates (or the number density of critical clusters). The underestimate by the CNT is more significant at lower temperatures. On the other hand, the SP model gives a proper correction to the CNT prediction and achieves excellent agreement with all of the results of the MD simulations for both the low- and high- $T$ cases. This indicates that the SP model predicts the free energy of cluster formation $\Delta G_{i}$ (or the cluster integrals) with good accuracy for a wide range of temperatures. In the MD simulations, sizes of the critical clusters are smaller than 20 because of the high initial supersaturation ratios. In order to examine the transition to the scaling law by McGraw and Laaksonen, we must consider additional low- $S_{0}$ cases.

2. The sticking probability of vapor molecules onto clusters is also measured from the growth rate of stable clusters in the MD simulations of the present study. The sticking probability $\alpha$ decreases with the supersaturation ratio for a given temperature (Fig. 10(b)). Using the values of $\alpha$ obtained by the MD simulations, we can further increase the accuracy of the SP model (Fig. 11). The lowest $\alpha$ is $\sim 0.1$ for the ranges of the parameters adopted in the MD simulations, the sticking probability would be much smaller than 0.1 for the lower supersaturation ratios. For such low- $S$ cases, we must estimate $\alpha$ precisely for the evaluation of $J$. The sticking probability of low- $S$ should be examined in the future.

3. In all of the MD simulation runs, the clusters are first nucleated as supercooled liquid droplets, although the temperature is lower than the triple point. This would be because of the small sizes of the nuclei and heating by latent heat deposition. For the low-temperature cases of
$T \leq 0.4 \varepsilon / k$, crystallization of supercooled liquid nuclei is observed after the slowing of their growth. The details of the crystallization process of clusters should be investigated further in the future.

\section{ACKNOWLEDGMENTS}

We are grateful to Akio Kawano and anonymous reviewers for precious comments. T.Y. acknowledges the support from JSPS (21244011).

\section{APPENDIX A: THE RELATION BETWEEN THE CHEMICAL POTENTIAL AND THE CLUSTER INTEGRAL}

The partition function $Z_{N}(T, V, N)$ of a system composed of $N$ identical molecules of mass $m$ is given by

$$
Z_{N}(T, V, N)=\left(\frac{2 \pi m k T}{h^{2}}\right)^{3 N / 2} j(T)^{N} Q_{N},
$$

where $T$ and $V$ are the temperature and volume of the system, respectively, $j(T)$ is the partition function of a single molecule with respect to its internal degrees of freedom, and $Q_{N}$ is a configuration integral given by

$$
Q_{N}=\frac{1}{N !} \int \cdots \int e^{-U / k T} d \mathbf{r}_{1} \cdots d \mathbf{r}_{l} .
$$

In the above equation, $U$ is the total potential energy of the system, that is, a sum of the interaction potentials $u_{j l}(j>l)$ for all pairs of molecules in the system. Here, it is assumed that the potential is independent of the internal motions of the molecules, and that the force acting between molecules is the central force, such as L-J force.

The cluster integral $b_{i}$ for $i$-mers is defined as

$$
b_{i}=\frac{1}{i ! V} \int \ldots \int \sum_{\substack{i \geq j>l \geq 1 \\ \text { sum over all products } \\ \text { consistent with a single cluster. }}}\left(e^{-u_{j l} / k T}-1\right) d \mathbf{r}_{1} \cdots d \mathbf{r}_{i} \text {, }
$$

where $V$ is the volume of the system and $u_{j l}$ is the interaction potential between monomers labeled $j$ and $l$ composing an $i$ mer. Here, the product is taken for a pair of monomers bonded with each other in a cluster of a given structure, and the sum is taken over all possible structures of clusters of $i$ ( $i$-mers). For example, $b_{1}=1$, and $-b_{2}$ is equal to the second virial coefficient $B_{2}$ (see Eq. (B3) in Appendix B). Note that each cluster integral is a function of temperature.

Using the cluster integrals defined by Eq. (A3), the configuration integral $Q_{N}$ is expanded as ${ }^{53}$

$$
Q_{N}=\sum_{\substack{\left\{m_{i}\right\} \\ \sum i m_{i}=N}} \prod_{i=1}^{N} \frac{\left(V b_{i}\right)^{m_{i}}}{m_{i} !}
$$

where the sum is taken all over the ways of assigning $N$ molecules to a set of cluster sizes $\left\{m_{i}\right\}$ under the condition of $\sum i m_{i}=N$. On the other hand, the grand partition 
function $\Xi$ for a given chemical potential $\mu$ is given by

$$
\begin{gathered}
\Xi(T, \mu, V)=\sum_{N=0}^{\infty} e^{N \mu / k T} Z_{N} \\
=\exp \left[V \sum_{i=1}^{\infty} b_{i} e^{i \mu / k T}\left(\frac{2 \pi m k T}{h^{2}}\right)^{3 i / 2} j(T)^{i}\right] .
\end{gathered}
$$

From the relation $P V=k T \ln \Xi$, the total pressure $P$ and the density $\rho$ of the imperfect gas are expanded with the use of the cluster integrals $b_{i}$ as

$$
P=k T \sum_{i=1}^{\infty} b_{i}\left(\frac{P_{1}}{k T}\right)^{i}
$$

and

$$
\rho=m \sum_{i=1}^{\infty} i b_{i}\left(\frac{P_{1}}{k T}\right)^{i},
$$

respectively, where we use the following expression of the chemical potential:

$$
\frac{\mu}{k T}=\ln \left[\frac{P_{1}}{k T}\left(\frac{h^{2}}{2 \pi m k T}\right)^{3 / 2} j^{-1}\right] .
$$

We can obtain another expression for the equation of state by considering an imperfect gas to be an ideal gas mixture of monomers and $i$-mer clusters. Using the equilibrium number densities of clusters and monomers, $n_{\mathrm{e}}(i)$, the total pressure and the density are expressed as

$$
P=k T \sum_{i=0}^{\infty} n_{\mathrm{e}}(i) \text {. }
$$

and

$$
\rho=\sum_{i=0}^{\infty} i m n_{\mathrm{e}}(i)
$$

The two kinds of expressions of Eqs. (A7) and (A10) for $P$ and Eqs. (A8) and (A11) for $\rho$ should be equivalent to each other. Actually, since $n_{\mathrm{e}}(i) \propto P_{1}^{i}$ (see Eq. (5)), $P$ and $\rho$ are expressed in the terms of a power series of $P_{1}$ in both the latter and former approaches. Comparing the terms that are proportional to $P_{1}^{i}$ in both approaches, we obtain

$$
n_{\mathrm{e}}(i)=b_{i}\left(\frac{P_{1}}{k T}\right)^{i} .
$$

Note that $n_{e}(i)$ should be positive in the latter approach. On the other hand, the second cluster integral $b_{2}$ (the second virial coefficient) is negative (positive) at temperatures above the critical point. This indicates that the assumption of an ideal gas mixture in the latter approach is valid only below the critical point.

Finally, substituting Eq. (7) into Eq. (A12), we obtain the following relation between the chemical potential $\mu_{i}^{0}(T)$ and the cluster integral $b_{i}(T)$ :

$$
\mu_{i}^{0}-i \mu_{\mathrm{sat}}=-k T \ln \left\{b_{i}\left(\frac{P_{\mathrm{sat}}}{k T}\right)^{i-1} \frac{P_{\mathrm{sat}}}{P_{0}}\right\} .
$$

\section{APPENDIX B: THE EMPIRICAL FORMULAE OF THE THERMODYNAMIC QUANTITIES}

For surface tension of the super-cooled L-J liquid, we used the formula given by 57

$$
\sigma_{s}=2.942 \times\left(1-\frac{T}{T_{\mathrm{c}}}\right)^{1.303},
$$

where the critical temperature $T_{\mathrm{c}}$ is $1.312 \varepsilon / k$. The saturated vapor pressure $P_{\text {sat }}$ for the super-cooled L-J liquid is given by

$$
P_{\text {sat }}=\frac{\varepsilon}{\sigma^{3}} \exp \left\{A-\frac{C \varepsilon}{k T}\right\},
$$

where the coefficients are $A=3.24157$ and $C=6.91117 .^{54}$ Although this parameterization is obtained based on the MD simulations ${ }^{54}$ in the limited range of temperatures 0.7 $\leq k T / \varepsilon \leq 1.127$, it agrees well with the numerical results of MD simulations ${ }^{57}$ for $0.5 \leq k T / \varepsilon \leq 1.2$ and those of MC simulations $^{58}$ for $0.25 \leq k T / \varepsilon \leq 0.875$. It should be noted that there are some uncertainties in the values of vapor pressure (of about a factor 2) and in surface tension (of about 10\%) at the low temperature of $k T \sim 0.2 \varepsilon$.

The second virial coefficient $B_{2}$ is given by

$$
B_{2}=2 \pi \int_{0}^{\infty}\left(1-e^{-\frac{u_{i j}}{k T}}\right) r^{2} d r,
$$

where $r$ is the distance between two molecules. In the MD simulations, the potential in the simulations is truncated at $5 \sigma$. The resulting virial coefficients are about $1 \%$ lower, but this difference is negligible for the prediction of the nucleation rate in the SP model.

According to Kalikmanov et al., ${ }^{47}$ the bulk density of the argon liquid is given by

$$
\begin{aligned}
\rho_{m}= & 39.948 \times 10^{-3}\left(13.29+24.49248 f^{0.35}\right. \\
& +8.155083 f) \mathrm{m} \sigma^{-3} \mathrm{~g} \mathrm{~cm}^{-3}
\end{aligned}
$$

with

$$
f=1-\frac{T}{150.633[\mathrm{~K}]} .
$$

We used these formulae with changing the L-J units $\sigma$ and $\varepsilon$.

\footnotetext{
${ }^{1}$ M. Volmer and A. Weber, Z. Phys. Chem. 119, 277 (1926).

${ }^{2}$ V. R. Becker and W. Döring, Ann. Phys. 24, 719 (1935).

${ }^{3}$ J. B. Zel'dovich, J. Exp. Theor. Phys. 12, 525 (1942).

${ }^{4}$ J. Feder, K. C. Russell, J. Lothe, and G. M. Pound, Adv. Phys. 15, 111 (1966).

${ }^{5}$ J. L. Schmitt, G. W. Adams, and R. A. Zalabsky, J. Chem. Phys. 77, 2089 (1982).

${ }^{6}$ J. L. Schmitt, R. A. Zalabsky, and G. W. Adams, J. Chem. Phys. 79, 4496 (1983).

${ }^{7}$ G. W. Adams, J. L. Schmitt, and R. A. Zalabsky, J. Chem. Phys. 81, 5074 (1984).

${ }^{8}$ A. Dillmann and G. E. A. Meier, J. Chem. Phys. 94, 3872 (1991).

${ }^{9}$ D. W. Oxtoby, J. Phys.:Condens. Matter 4, 7627 (1992).

${ }^{10}$ C. F. Delale and G. E.A. Meier, J. Chem. Phys. 98, 9850 (1993).

${ }^{11}$ Y. Viisanen, R. Strey, and H. Reiss, J. Chem. Phys. 99, 4680 (1993).

${ }^{12}$ D. Wright, R. Caldwell, C. Moxely, and M. S. El-Shall, J. Chem. Phys. 98, 3356 (1993).

${ }^{13}$ A. Laaksonen, I. J. Ford, and M. Kulmala, Phys. Rev. E 49, 5517 (1994).

${ }^{14}$ Y. Viisanen and R. Strey, J. Chem. Phys. 101, 7835 (1994).

${ }^{15}$ K. Hämeri and M. Kulmala, J. Chem. Phys. 105, 7696 (1996).
} 
${ }^{16}$ D. Kane and M. S. El-Shall, J. Chem. Phys. 105, 7617 (1996).

${ }^{17}$ I. J. Ford, Phys. Rev. E 56, 5615 (1997).

${ }^{18}$ M. P. Anisimov and P. K. Hopke, J. Chem. Phys. 115, 810 (2001).

${ }^{19}$ I. Kusaka, A.-G. Wang, and J. H. Seinfeld, J. Chem. Phys. 108, 3416 (1998).

${ }^{20}$ K. Yasuoka and M. Matsumoto, J. Chem. Phys. 109, 8451 (1998).

${ }^{21}$ K. Yasuoka and M. Matsumoto, J. Chem. Phys. 109, 8463 (1998).

${ }^{22}$ P. R. ten Wolde and D. Frenkel, J. Chem. Phys. 109, 9901 (1998).

${ }^{23}$ I. Kusaka and D. W. Oxtoby, J. Chem. Phys. 110, 5249 (1999).

${ }^{24}$ K. J. Oh and X. C. Zeng, J. Chem. Phys. 110, 4471 (1999).

${ }^{25}$ B. Senger, P. Schaaf, D. S. Corti, R. Bowles, D. Pointu, J.-C. Voegel, and H. Reiss, J. Chem. Phys. 110, 6438 (1999).

${ }^{26}$ P. R. ten Wolde, M. J. Ruiz-Montero, and D. Frenkel, J. Chem. Phys. 110, 1591 (1999).

${ }^{27}$ K. Laasonen, S. Wonczak, R. Strey, and A. Laaksonen, J. Chem. Phys. 113, 9741 (2000)

${ }^{28}$ K. J. Oh and X. C. Zeng, J. Chem. Phys. 112, 294 (2000).

${ }^{29}$ S. Tanimura, K. Yasuoka, and T. Ebisuzaki, J. Chem. Phys. 112, 3812 (2000).

${ }^{30}$ H. Vehkamäki and I. J. Ford, J. Chem. Phys. 112, 4193 (2000).

${ }^{31}$ B. Chen, J. I. Siepmann, K. J. Oh, and M. L. Klein, J. Chem. Phys. 115, 10903 (2001).

${ }^{32}$ P. Schaaf, B. Senger, J.-C. Voegel, R. K. Bowles, and H. Reiss, J. Chem. Phys. 114, 8091 (2001).

${ }^{33}$ S. Yoo, K. J. Oh, and X. C. Zeng, J. Chem. Phys. 115, 8518 (2001).

${ }^{34}$ S. Toxvaerd, J. Chem. Phys. 115, 8913 (2001).

${ }^{35}$ S. Toxvaerd, J. Chem. Phys. 119, 10764 (2003).

${ }^{36}$ K. K. Tanaka, H. Tanaka, K. Kawamura, and K. Nakazawa, J. Chem. Phys. 122, 184514 (2005).

${ }^{37}$ H. Matsubara, T. Koishi, T. Ebisuzaki, and K. Yasuoka, J. Chem. Phys. 127, 214507 (2007).

${ }^{38}$ R. McGraw and A. Laaksonen, Phys. Rev. Lett. 76, 2754 (1996).
${ }^{39}$ R. McGraw and A. Laaksonen, J. Chem. Phys. 106, 5284 (1997).

${ }^{40}$ D. Reguera, R. K. Bowles, Y. Djikaev, and H. Reiss, J. Chem. Phys. 118, 340 (2003).

${ }^{41}$ D. Reguera and H. Reiss, Phys. Rev. Lett. 93, 165701 (2004).

${ }^{42}$ V. I. Kalikmanov, J. Chem. Phys. 124, 124505 (2006).

${ }^{43}$ J. Wedekind, J. Wölk, D. Reguera, and R. Strey, J. Chem. Phys. 127, 154515 (2007)

${ }^{44}$ J. Merikanto, E. Zapadinsky, A. Lauri, I. Napari, and H. Vehkamäki, J. Chem. Phys. 127, 104303 (2007).

${ }^{45}$ J. Merikanto, E. Zapadinsky, A. Lauri, and H. Vehkamäki, Phys. Rev. Lett. 98, 145702 (2007).

${ }^{46}$ B. Chen, H. Kim, S. J. Keasler, and R. B. Nellas, J. Phys. Chem. B 112, 4067 (2008).

${ }^{47}$ V. I. Kalikmanov, J. Wölk, and T. Kraska, J. Chem. Phys. 128, 124506 (2008).

${ }^{48}$ J. Wedekind, G. Chkonia, J. Wölk, R. Strey, and D. Reguera, J. Chem. Phys. 131, 114506 (2009).

${ }^{49}$ J. Julin, I. Napari, J. Merikanto, and H. Vehkamäki, J. Chem. Phys. 129, 234506 (2008).

${ }^{50}$ I. Napari, J. Julin, and H. Vehkamäki, J. Chem. Phys. 133, 154503 (2010).

${ }^{51}$ K. Yasuoka, M. Matsumoto, and Y. Kataoka, J. Chem. Phys. 101, 7904 (1994).

${ }^{52}$ G. Nagayama and T. Tsuruta, J. Chem. Phys. 118, 1392 (2003).

${ }^{53}$ J. E. Mayer and M. G. Mayer, Statistical Mechanics (Wiley, New York, 1940).

${ }^{54}$ A. Trokhymchuk and J. Alejandre, J. Chem. Phys. 111, 8510 (1999).

${ }^{55}$ B. Quentrec and C. Brot, J. Comput. Phys. 13, 430 (1973).

${ }^{56}$ W. Mattson and B. M. Rice, Comput. Phys. Commun. 119, 135 (1999).

${ }^{57}$ V. G. Baidakov, S. P. Protsenko, Z. R. Kozlova, and G. G. Chernykh, J. Chem. Phys. 126, 214505 (2007).

${ }^{58}$ J. C. Barret and A. P. Knight, J. Chem. Phys. 128, 086101 (2008). 